\title{
IMPROVEMENTS IN CALCULATION OF RENAL RESISTANCE TO BLOOD FLOW. CHARTS FOR OSMOTIC PRESSURE AND VISCOSITY OF BLOOD
}

\author{
BY HAROLD LAMPORT ${ }^{1}$
}

\begin{abstract}
(From the Department of Neurology, College of Physicians and Surgeons, Columbia University, and the Neurological Institute, New York City, and the Department of Physiology, Yale University School of Medicine, New Haven)
\end{abstract}

(Received for publication November 19, 1942)

Previously, we have offered formulas to determine the resistance to blood flow of the afferent and efferent arterioles of the kidney (1). They employed a modified Poiseuille's Law and were based especially on the concept of osmotic equilibrium in the glomerulus between the blood leaving the glomerular capillaries and the ultrafiltrate in Bowman's capsule. They utilized the inulin clearance as a measure of the rate of glomerular filtration and the diodrast clearance as a measure of the rate of renal plasma flow, as developed by Homer Smith and his coworkers $(2,3)$. For determining the osmotic pressure of blood both before and after glomerular filtration, we depended on the data of the Adairs and Greaves (4) for human serum diluted below normal, not having been able, as yet, to supplant them with observations on concentrated plasma.

The formulas for renal afferent and efferent arteriolar resistance were, respectively (1):

$$
\begin{gathered}
R_{A}=\frac{P_{M}-P_{o^{\prime}}-40}{H D} ; \\
R_{B}=\frac{(1-0.47 F)\left(P_{o^{\prime}}-P_{0}+10\right)}{H D} .
\end{gathered}
$$

Here $P_{M}$ is the mean of systolic and diastolic blood pressure in $\mathrm{mm} . \mathrm{Hg}, D$ is the effective renal plasma flow (diodrast clearance, usually), and $H$ is the reciprocal of (1-hematocrit). $F$ is the glomerular filtration fraction (the ratio of inulin to diodrast clearance), $P_{o^{\prime}}$ is the osmotic pressure in $\mathrm{mm}$. of $\mathrm{Hg}$ of the blood after concentration by glomerular filtration, and $P_{0}$ is the value before glomerular concentration. The osmotic pressures were given in terms of a formula which depended on the serum protein and filtration fraction, the use of which will be discussed later. The unit for $R_{A}$ and $R_{B}$ depends on the unit for $D$. Ordinarily, it will be $\mathrm{mm}$. $\mathrm{Hg}$ per

1 Now at Yale University School of Medicine, New Haven. cc. per minute per 1.74 square meters of body surface.

\section{Poiseuille's Law}

In our original use of Poiseuille's law, we followed Whittaker and Winton's (5) description of the viscosity of blood of varying hematocrit (1) in the pump-lung-limb of the dog. They found that pressure and flow were strictly proportional for Ringer's solution, but that a constant had to be subtracted from the pressure when whole blood was used. This constant was about $20 \mathrm{~mm}$. $\mathrm{Hg}$ for normal hematocrits but of course it decreased with the hematocrit so that for nearly zero hematocrit (plasma), it was not far from zero. At the time of the formulation of our equations for resistance, we were not aware of the similarity between Whittaker and Winton's in vivo findings for blood and the large body of observation in the field of physical chemistry concerning the flow of mixtures, emulsions, suspensions, and plastic solids. These facts are summarized in a monograph by Bingham (6) on which we draw. Similar conclusions are found elsewhere (7).

The flow-pressure curve of a plastic solid is identical in form with the almost linear curves for blood found by Whittaker and Winton. Bingham gives the relationship (p. 225, with modification) as:

Resistance $=\frac{\text { Perfusion pressure }- \text { Yield pressure }}{\text { Rate of flow } X \text { Viscosity }}$.

Apparently, part of the applied pressure is used in producing viscous flow and part in overcoming the internal friction of the red cells. As a whole, there is plastic flow. This equation would imply that no flow would occur at low values of pressure, but this is not actually true in plastic solids and Bingham offers various explanations for the observed continuance of slight flow at low pressures. (One of them is the same 
as Whittaker and Winton's suggestion in their discussion of the low apparent viscosity of blood in vivo.) Furthermore, this formula is not quite correct slightly above the yield pressure. It applies only to the linear portion of the pressureflow curve.

As has been indicated, we have offered no formula to express yield pressure in terms of the hematocrit, having had no basis on which to do so. Our formula used a constant quantity, 20 $\mathrm{mm}$. $\mathrm{Hg}$, obtained from Whittaker and Winton's findings for normal hematocrits, as the value for yield pressure. Bingham's analysis, however, enables us to make progress by using a variable rather than a static yield pressure.

He defines $f$ as the internal friction in the plastic in terms of shear. It is a constant, independent of the viscometer capillary's dimensions and is related to $P_{f}$, the yield pressure, as follows, where $r$ is the radius of the bore of the capillary viscometer and $L$ is its length:

$$
f=\frac{r}{2 L} P_{f}
$$

Also, where $A$ and $B$ are constants, independent of the viscometer and $H_{c}$ is the hematocrit,

$$
f=A+B H_{c},
$$

since $f$ is linearly related to the volume concentration of the suspended substance (6). Then,

$$
P_{f}=\frac{2 L}{r}\left(A+B H_{c}\right) \text {. }
$$

Since $P_{f}$ is nearly zero when $H_{c}$ is zero (if it is not actually zero-back-pressure due to edema and tissue elasticity may cause an artifact), we can set $A=0$. Then,

$$
P_{f}=\frac{2 L}{r} B H_{c} \text {. }
$$

In this application, we have imagined the portion of the blood vascular system we are considering to be replaced by an ideal viscometer of uniform bore (radius $r$ ), length $L$, and resistance to blood flow $T$. If we always choose the bore of our ideal equivalent viscometer so that it is the same for the same degree of vasoconstriction, and vary its length to adjust for the different organs and regions, the resistance of which is being studied, $r$ will represent the degree of vaso- dilatation and $1 / r$ the degree of vasoconstriction. Such a model indicates that the yield pressure in the study of a particular organ is proportional to the hematocrit and to the degree of vasoconstriction.

Now, Bingham shows that for plastic solids, as for viscous fluids, the resistance is proportional to the length and inversely proportional to the fourth power of the radius of the capillary viscometer. Thus:

$$
T=L / b^{4} r^{4} .
$$

Here $b$ is a constant. We then have:

$$
P_{f}=b B H_{c}(T / L)^{t} \text {. }
$$

The temptation, at this point, is to evaluate the unknown quantity, $b B / L^{t}$, by using Whittaker and Winton's data; set $P_{f}=20 \mathrm{~mm}$. Hg, $H_{c}=\frac{1}{2}$; calculate $T$ (the resistance of the hindlimb of the dog) and insert it in the above formula. But such a method, while entirely legitimate for the hind-limb preparation, is not applicable to the kidney, since both $L$ and $T$ are dependent on the region in which they are measured. What is true of the hind-limb does not apply to the kidney, so far as $L$ and $T$ are concerned. Actually, the replacement of $r$ by $T$, et cetera, in the expression for $P_{f}$ is not really an improvement unless the expression can be evaluated by means of a kidney pressure-flow curve, similar to Whittaker and Winton's for the hind-limb of the dog, the intercept of which would give us the yield pressure for a given degree of vasoconstriction (flow $=0$; pressure $=P_{f}$ ). The possibility of obtaining such pressure-flow curves for the kidney seems remote. That organ, the vessels of which appear to react autonomously to changes in blood pressure, would not maintain constancy of vessel size (constant resistance) while the pressure was being varied. Clearly, an approximation to yield pressure in the kidney must be used, if it is justifiable.

Our last expression for $P_{f}$, above, shows that, for a given site ( $L$ constant), $P_{f}$ varies as the fourth root of the resistance to blood flow (when on the linear portion of the pressure-flow curve). That is to say, $P_{f}$ is not very sensitive to changes in resistance. Thus, a 50 per cent increase in resistance entails an increase in $P_{f}$ of 11 per cent. If $P_{f}$ is $20 \mathrm{~mm}$. $\mathrm{Hg}$, its approximate value in the 
leg preparation with normal hematocrit, a 50 per cent increase in resistance would augment $P_{\boldsymbol{f}}$ by about $2 \mathrm{~mm}$. Hg. Such a quantity seems unimportant at the present stage of biological precision in renal physiology. The greatly increased difficulty in computation resulting from modifying the yield pressure by the fourth root of the resistance would hardly compensate for the slight improvement except in highly abnormal circumstances. $^{2}$ We therefore can neglect for ordinary physiological purposes the variable effect on yield pressure of the resistance, retaining solely the hematocrit.

We continue to use $20 \mathrm{~mm}$. $\mathrm{Hg}$ for the yield pressure, the figure obtained from Whittaker and Winton when the hematocrit is normal (0.43). Although this may not be the best value for the kidney, it is the best available and is probably not far wrong, since we have seen that the yield pressure is relatively insensitive to changes in resistance. While the kidney has a high rate of blood flow for its size, compared to muscle (the hind limb of the dog), Whittaker and Winton's preparations were studied in a state of dilatation so that comparison between the two sites may be justified as an approximation within our present limits of precision. ${ }^{3}$ We therefore write for $P_{f}$ the following expression (based on $P_{f}=20$, when $H_{c}=0.43$ ):

$$
P_{f}=46 H_{c} .
$$

Since the blood pressure is reduced to the usual capillary pressure, in passing through the kidney, in two stages - the afferent and the efferent arterioles-rather than one, it is advisable to allocate $P_{f}$ between the afferent and efferent arteriolar resistances, neglecting the small capillary and venous terminal portion $\left(R_{V}\right)$. Our previous formulas for $R_{A}$ and $R_{E}$, the afferent and efferent arteriolar resistances, were obtained by alloting all of the fixed yield pressure $(20 \mathrm{~mm}$.

\footnotetext{
2 Should evidence appear indicating the value of such a modification, a fairly good approximation may be made by using

$P_{f}=$ Constant $\times H_{c} \frac{\left(\text { Perfusion pressure }- \text { Constant } \times H_{c}\right)^{t}}{\text { Flow }}$.

3 The relatively low resistance of the kidney to blood flow also suggests that the linear portion of the pressureflow curve is normally present.
}

$\mathrm{Hg}$ ) to the afferent arteriolar resistance. ${ }^{4}$ Under this circumstance, $R_{A}$ is slightly larger than $R_{E}$ (9). If $P_{f}$ is distributed between $R_{A}$ and $R_{E}$, as now seems advisable, this preponderance of $R_{A}$ over $R_{E}$ is enhanced. Whether the afferent arterioles are of greater length or greater constriction (or both) than the efferent arterioles is not clear, but in view of the few millimeters of mercury difference in yield pressure between one possibility and the other, at this time, for lack of better information, an equal partition of $\boldsymbol{P}_{\boldsymbol{f}}$ between $R_{A}$ and $R_{E}$ seems wise.

Accordingly, we have:

$$
\begin{aligned}
& R_{A}=\frac{\text { Perfusion pressure afferent arterioles }-\frac{1}{2} P_{f}}{\text { Rate of flow } \times \text { Viscosity }} ; \\
& R_{E}=\frac{\text { Perfusion pressure efferent arterioles }-\frac{1}{2} P_{f}}{\text { Rate of flow } \times \text { Viscosity }}
\end{aligned}
$$

Since $\frac{1}{2} P_{f}=23 H_{c}$, the formulas become:

$$
\begin{aligned}
& R_{A}=\frac{P_{M}-P_{0^{\prime}}-23 H_{c}-20}{H D} ; \\
& R_{E}=\frac{(1-0.47 F)\left(P_{o^{\prime}}-P_{o}-23 H_{c}+10\right)}{H D} .
\end{aligned}
$$

While these formulas supersede the previous ones, it is unlikely that they would seriously modify the results obtained from the older version, being a refinement based on a more precise value for yield pressure and its allocation to both afferent and efferent arteriolar resistances.

\section{Post-arteriolar renal resistance to blood flow}

The formulas developed have been limited to arterial and arteriolar resistance; they did not include the resistance of the kidney to blood flow after the glomerular filtrate had been reabsorbed in the capillaries and the blood flowed on into the renal vein, outside the kidney. But the same methods can be applied to evaluate this post-arteriolar resistance.

It will be, as before, the perfusion pressure less the yield pressure, divided by the rate of blood flow times viscosity. Or, where $R_{V}$ is this last portion of renal resistance referred to the sub-

\footnotetext{
1 The effect of glomerular capillary resistance, which has been included in the expression for afferent arteriolar resistance, has been assayed in the published report (1), though it was probably not available to Shannon, who no doubt depended on an earlier manuscript version, when noting its absence in a recent review of renal physiology (8).
} 
ject's own blood as perfusion standard (viscosity $=1$ ), and $P_{V}$ is the pressure of the renal vein in $\mathrm{mm}$. of $\mathrm{Hg}$, we have:

$$
R_{\nabla}=\frac{\begin{array}{c}
\text { (Initial pressure }- \text { Terminal pressure) } \\
- \text { Yield pressure }
\end{array}}{H D} .
$$

Because of the relatively slight resistance after the arterioles, it seems inadvisable to allocate any of the previously discussed yield pressure to $R_{V}$, so that this term drops out.

$$
R_{V}=\frac{\text { Initial pressure }-P_{V}}{H D}
$$

This initial pressure is clearly the same as the terminal pressure for efferent arteriolar resistance, which was $P_{o}+P_{R}$, that is-the osmotic pressure of the systemic blood plus the renal interstitial pressure, considered to be $20 \mathrm{~mm}$. $\mathrm{Hg}$. We therefore have:

$$
R_{V}=\frac{P_{o}-P_{V}+20}{H D} .
$$

Such a formula is, of course, an approximation. It suggests, since the numerator is constant, that the capillaries and venules dilate with increased blood flow so as to reduce the resistance in proportion to the increased flow. Actually, this is not completely true. Our definition of efferent arteriolar resistance, by using a fixed terminal pressure, is more functional than anatomical in scope. With a marked increase in blood flow, for example, it is likely that this fixed pressure point shifts along the capillaries, towards the venous end, so that anatomically a portion of what was originally included in $R_{V}$ would be shifted to $R_{E}$. So long as the limitations of our definitions of $R_{E}$ and $R_{V}$ are explicit and remembered no harm can result.

Total renal resistance now can be defined:

$$
R_{K}=R_{A}+R_{E}+R_{V} .
$$

In this way, total resistance from the renal artery to the renal vein has been evaluated.

Osmotic pressure affected by $A: G$ ratio

The formulas for renal resistance depend on an expression for the osmotic pressure of blood, derived from one offered by the Adairs and Greaves (4) from measurements on serum of a particular albumin-globulin ratio. We made no correction for $A$ : $G$ ratios differing from theirs of 2.20. While the discrepancy thereby resulting is usually not large, we have been able to minimize it by applying an empirical formula of Wies and Peters (10) for the osmotic pressure of a large range of $A: G$ ratios in human sera. They found that albumin was 2.66 times as effective as globulin in elevating osmotic pressure in the range studied.

Let us call the concentration of serum proteins $\bar{S}$ in grams per $100 \mathrm{cc}$. with an A: G ratio of $B$. Let $S$ be the concentration of serum protein, in grams per $100 \mathrm{cc}$., which would be required of the Adairs' and Greaves' 2.20 A : G ratio serum (our standard) to have the same osmotic pressure as the sample with the $B$ ratio. Let $g$ be the number of millimeters of mercury osmotic pressure contributed per gram globulin in serum.

We then have:

1 gram globulin produces $\mathrm{g} \mathrm{mm}$. $\mathrm{Hg}$ osmotic pressure.

1 gram albumin produces $2.66 \mathrm{~g} \mathrm{~mm}$. $\mathrm{Hg}$ osmotic pressure.

1 gram protein of $2.2 \mathrm{~A}: \mathrm{G}$ ratio contains 2.2/3.2 grams albumin, and 1/3.2 grams globulin.

1 gram protein of $B \quad A: G$ ratio contains $\frac{B}{B+1}$ grams albumin and $\frac{1}{B+1}$ grams globulin.

1 gram protein of $2.2 \mathrm{~A}: \mathrm{G}$ ratio has an osmotic pressure of

$$
\frac{2.2}{3.2} \times 2.66 g+\frac{1}{3.2} \times g .
$$

1 gram protein of $B$ A : $\mathrm{G}$ ratio has an osmotic pressure of

$$
\frac{B}{B+1} \times 2.66 g+\frac{1}{B+1} \times g \text {. }
$$

It is therefore clear that 1 gram of protein of $A$ : $G$ ratio $B$ can be replaced in computing its osmotic pressure by

$$
\begin{aligned}
\left(\frac{B}{B+1} \times 2.66 g\right. & \left.+\frac{1}{B+1} \times g\right) \\
& \div\left(\frac{2.2}{3.2} \times 2.66 g+\frac{1}{3.2} \times g\right)
\end{aligned}
$$

grams of protein of $2.20 \mathrm{~A}: \mathrm{G}$ ratio. That is to 
RENAL RESISTANCE. OSMOTIC PRESSURE AND VISCOSITY OF BLOOD

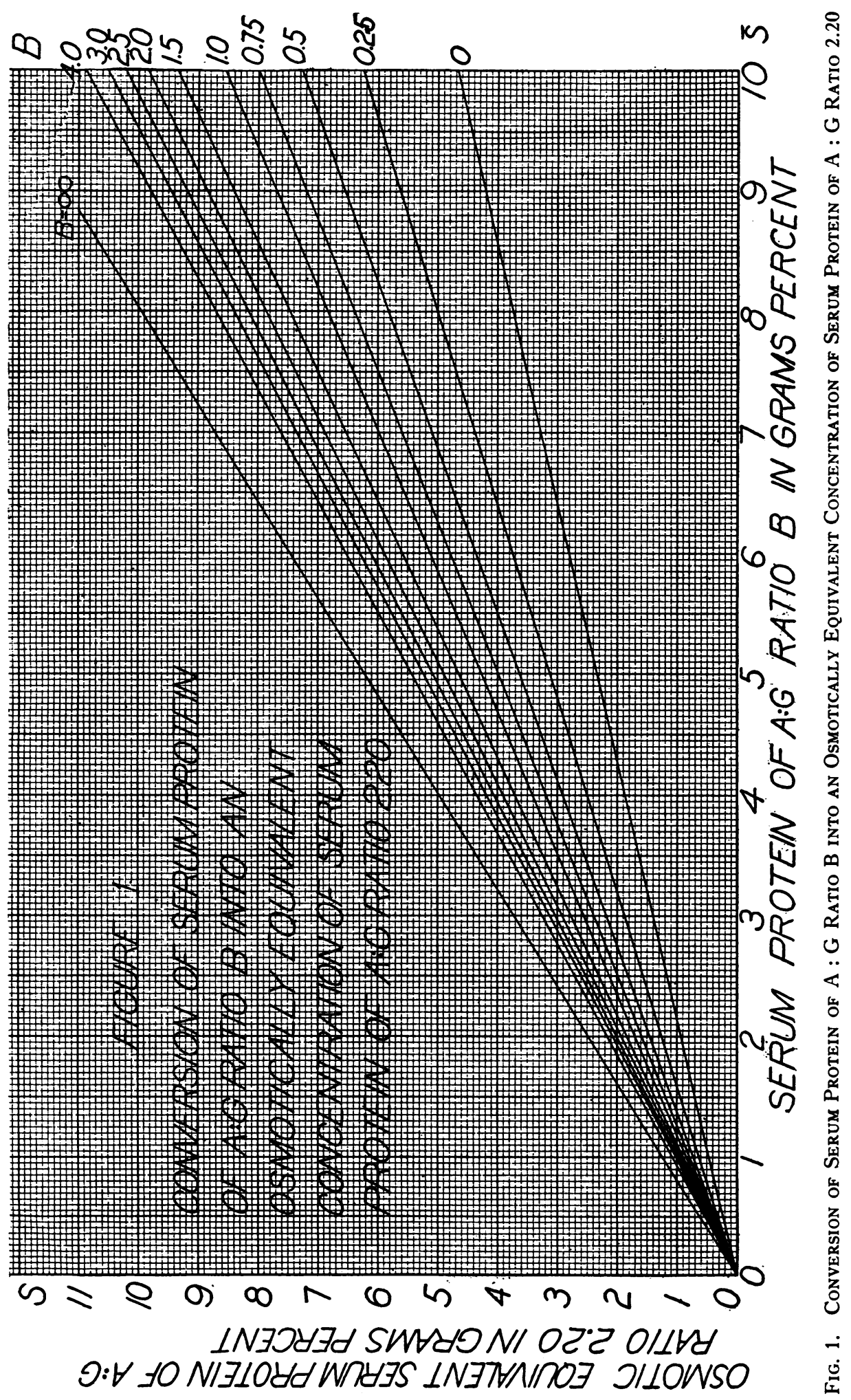


say:

Or,

$$
S=\frac{1+2.66 B}{1+B} \times \frac{3.2}{1+2.2 \times 2.66} \bar{S} .
$$

$$
S=\frac{0.467+1.243 B}{1+B} \bar{S} .
$$

This conversion formula is represented graphically in Figure 1, whereby we obtain the osmotic equivalent in terms of serum protein of $A: G$ ratio 2.20 for sera of varying $A: G$ ratio $B$.

The osmotic pressure in $\mathrm{mm}$. of $\mathrm{Hg}$ of plasma, at blood temperature, where the serum protein has the A : G ratio of 2.20 , our $S$, is (1):

$$
P_{o}=\frac{2.34 S}{1-0.0542 S} \text {. }
$$

In the glomerulus, a protein-free filtrate leaves the blood plasma, which is thereby concentrated. The fraction of plasma filtered off as glomerular filtrate $(F)$ is measured by the ratio of inulin clearance (glomerular filtration rate) to diodrast clearance (rate of effective renal plasma flow). The osmotic pressure of the plasma leaving the glomerulus $\left(P_{o^{\prime}}\right)$ is given by the formula:

$$
P_{o^{\prime}}=\frac{2.34 S}{1-0.0542 S-F} .
$$

This formula, for constant values of $P_{o^{\prime}}$, is represented by straight lines relating $S$ and $F$ and is shown in Figure 2. It is clear that when $F$ is set equal to zero, $P_{o}$, becomes identical with

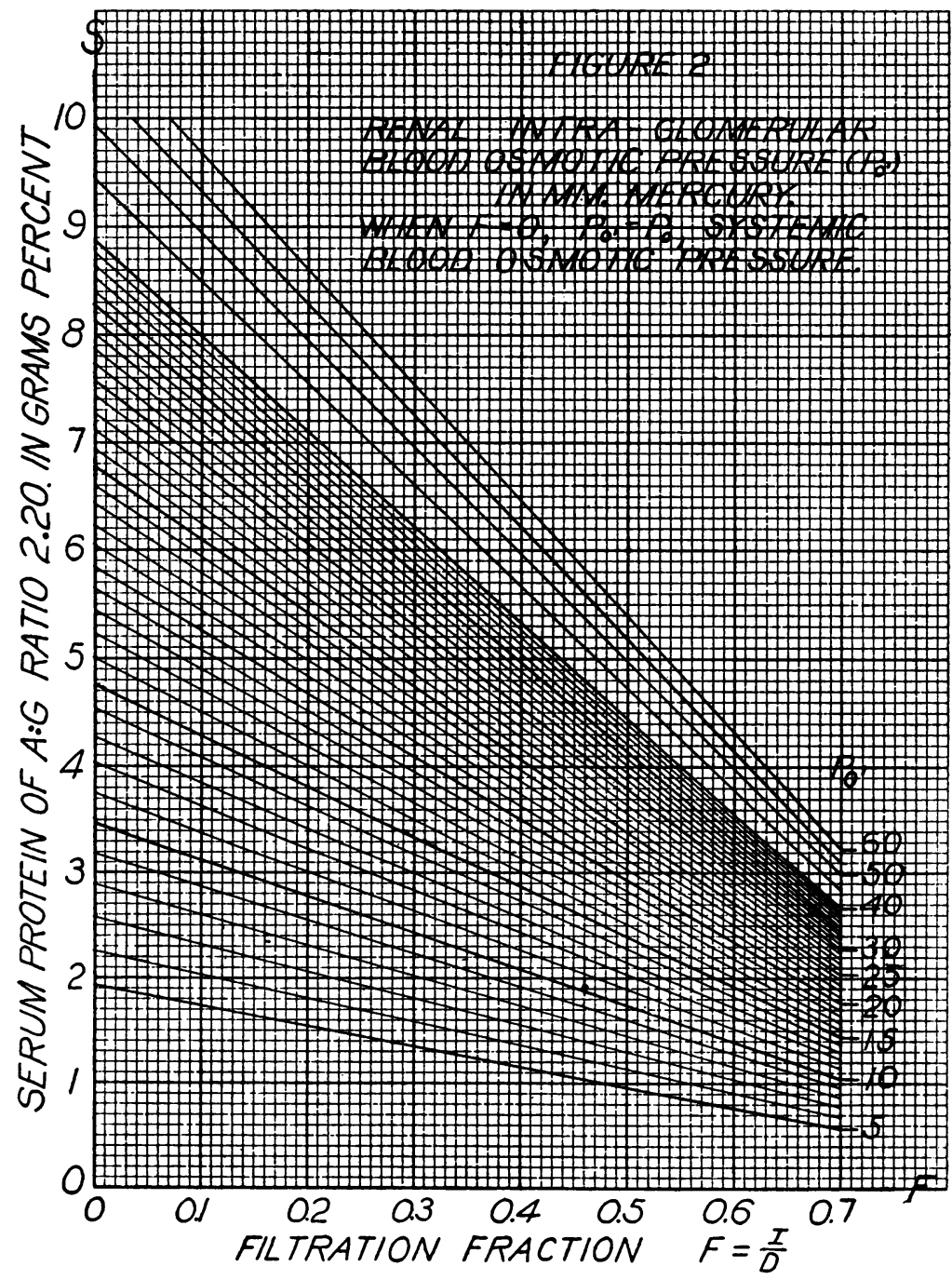

Fig. 2. Renal Intra-Glomerular Blood Osmotic Pressure 
$P_{0}$ (the osmotic pressure of the systemic blood). Thus, for a given $S$ and $F$, Figure 2 can be used to determine the corresponding values of both $P_{o}$ and $P_{\circ}$. The value of $S$, of course, would have come from Figure 1 by the insertion of the observed quantities $\bar{S}$ and $B$, the concentration of serum protein and its $A: G$ ratio.

An actual example will illustrate the use of these charts. If the serum protein is 6.0 grams per $100 \mathrm{cc}$. with an $A: G$ ratio of 1.5 , from Figure 1 we find that the osmotically equivalent protein concentration with an A : G ratio of 2.20 is 5.6. This value for $S$, in Figure 2, is found to give a value for $P_{0}$ (when $F=0$ ) of 18.9 and, if $F=0.2$, for example, a value for $P_{0}$, of 26.4. These quantities would then be utilized in the resistance formulas.

\section{VISCOSITY}

The present use of the resistance formulas has involved either the comparison of the subject with himself as control, after a brief interval for the experimental procedure, or the assumption that little change in blood viscosity has occurred $(1,11)$. In certain instances, where the hematocrit and serum protein are different and still a comparison of renal resistance is desired, the formulas may not be applicable unless their strict meaning is remembered.

As derived, $R_{A}$ and $R_{E}$ refer to the resistance of the afferent and efferent arterioles to the flow of the subject's own blood taken as the viscosity standard. If his blood changes, the values of $R_{A}$ and $R_{E}$ will refer to the changed blood as standard. Such a definition has real physiological value, since our interest in measuring resistance is certainly in part prompted by the desire to understand how much flow any given pressure will produce of the subject's own blood, irrespective of its viscosity. Thus, if the blood viscosity were halved while $R_{A}$ and $R_{E}$ were found to be unchanged, we should be able to say that, for the same pressure, the same rate of blood flow through the arterioles would persist. Such a condition, however, could be attained only by constriction of the arterioles. In the hydraulic sense, referred to some arbitrary unit, the resistance of the arterioles in our example must certainly have increased, when the blood viscosity was halved, to prevent increase in renal blood flow. We see, then, that the "hydraulic" resistance is necessary, if blood viscosity shifts, and we are interested in knowing the change produced in the calibre of the renal arterioles.

We are in a position to make this correction since we have a tentative expression for whole blood viscosity in terms of serum protein $(S)$ and the hematocrit $(1 / H=1$-hematocrit) (1). Let us call $U$ the viscosity of whole blood and $R_{a}$ and $R_{e}$ the afferent and efferent "hydraulic" arteriolar resistances referred to a fixed viscosity perfusion standard as compared with our present "individual" formulations, $R_{A}$ and $R_{E}$, which refer to the individual's own blood as viscosity standard. The small and large letter subscripts elsewhere will correspond analogously. We then have (1):

$$
R_{a}=R_{A} / U ; \quad R_{e}=R_{E} / U ;
$$

and where total "hydraulic" arteriolar resistance is $R_{r}$,

$$
R_{r}=R_{a}+R_{\mathrm{c}}=R / U
$$

where $R=R_{A}+R_{B}$. And similarly for the post-arterial resistance:

$$
R_{v}=R_{V} / U ; \quad R_{k}=R_{K} / U
$$

Since the relative change in blood viscosity as a result of glomerular hemoconcentration is already included in the expression for $R_{E}$, we need for $U$ only systemic blood viscosity, as previously offered (1).

$$
\begin{aligned}
& U=\text { plasma viscosity } \times(0.27+0.983 H) . \\
& U=(0.60+0.204 S)(0.27+0.983 H) .
\end{aligned}
$$

Or,

$$
U=(1+0.34 S)(0.162+0.590 H) \text {. }
$$

While this formula can be used to change $R_{A}$, and the other quantities, into $R_{a}, R_{e}$, and the corresponding analogues by computation, as shown above, the labor can be reduced by using Figure 3 , where, for known values of serum protein and $H$ (the hematocrit constant), the viscosity $U$ is given. In this way, values for "hydraulic" resistance of the renal arterioles and the rest of renal resistance can be obtained, which give a notion of the calib:e of these vessels, irrespective of the viscosity of the blood coursing through them. 


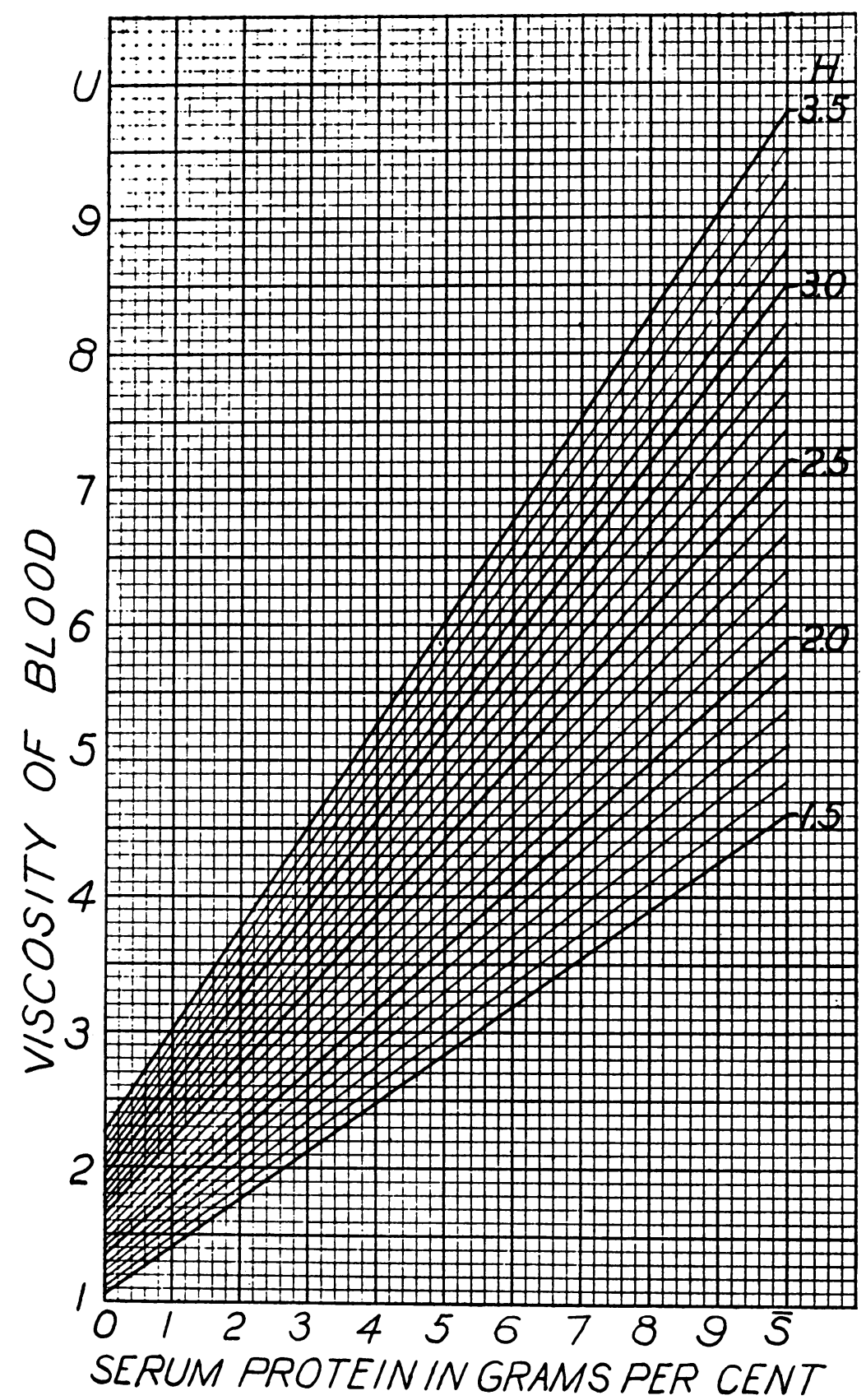

Fig. 3. Viscosity of Blood in Terms of Serum Protein and Hematocrit $H=1 /(1$ - hematocrit $)$.

\section{DISCUSSION}

The propriety of a mathematical treatment of renal resistance to blood flow can be questioned at the present time because of its dependence on "certain absolute values" to 'obtain a quantitative expression (8). Such c iticism is fundamentally unsound when it is used to support instead a non-mathematical treatment. The non-mathematical (or dialectic) method is concerned with the very same controlling quantities as the mathematical ones, but can achieve what appears to be a wider range of application and less dependence on these "absolute values" only by implicit neglect of the limitations which are 
inherent in the basic renal relationships. By stating premises concretely and limiting them where necessary, reasoning along mathematical lines can offer results, the underlying postulates of which are clear. A literary treatment simply obscures these facts and can lead to incorrect conclusions $(9,11,12)$.

The special virtue of algebraic expressions is the neat way in which the influences of many variables can simultaneously be permitted to interreact. Our minds, unaided by such tools, cannot be expected to assess the relative influence on resistance of changes in blood viscosity, blood pressure, blood flows, and so on. Far from assuming fixed afferent and efferent anteriolar resistances (8), wide variability of both is to be expected and obtained; the mode of derivation of the formulas does not presume fixity. This was clearly indicated in the initial application of these methods to renal resistance in man during spinal anesthesia, where changes of both sets of arterioles were routinely seen (1).

Nonetheless, because of the formulas' flexibility, and because it had previously been reported that in man, during the change from renal ischemia due to adrenalin to the renal hyperemia induced by pyrogens, the main shifts are in the efferent arterioles alone (3), it was deemed advisable to determine the effects of a fixed afferent or efferent set of arterioles. Our study indicated that, in this physiological range, both sets of arterioles, rather than only one, participated (11).

"The glomerular filtration rate cannot in theory, or in practice, approach zero at high renal blood flows . . . ," if the afferent arterioles and systemic blood pressure are fixed, according to Shannon (8). Yet, if we visualize our ideal nephron in this condition, we can see that, as efferent arteriolar resistance is progressively reduced, the drop in blood pressure along the efferent arterioles from glomerulus to peritubular capillaries must also progressively decrease, while blood flow, because of the consequent reduction in total renal resistance, rises. That is to say, as blood flow increases, glomerular intra-capillary pressure falls until, if carried to a theoretical (not physiological) extreme, it will be the same as peritubular capillary pressure and will be inadequate to form a glomerular filtrate. This is the meaning of the extreme foot of the curve pre- viously reported (11). It is not meant to be a physiological occurrence, since our formulas were carefully limited to physiological ranges so as to prevent drawing such impractical conclusions. We thus see that our ideal nephron, when carried past its well defined range, works reasonably, although ceasing to be applicable to biological problems.

That the calibre of arterioles, and therefore their resistance, varies with the pressure of the blood inside them (8) does not interfere with our deductions. Our "individual resistance," elaborated above, refers to the resistance to flow of the individual's own blood, and imposes no restrictions on the response of the arteriole to the pressure of the blood within it. "Hydraulic resistance," as we have used it, permits some conclusions concerning the relative calibre of the vessels, but again without neglect of their response to internal pressure.

\section{SUMMARY}

The similarity between blood flow and the flow of plastic solids, as described by Bingham, is used to substitute a variable in place of a static value for the yield pressure in our application of Poiseuille's Law to the kidney.

An expression for post-arteriolar resistance (defined precisely) is added to those for arteriolar resistance so that total renal resistance to blood flow can be computed, if desired.

A method is elaborated by which serum protein of any albumin-globulin ratio can be quickly converted by means of a chart (or formula) into an osmotically equivalent concentration of serum protein of $A: G$ ratio 2.20 .

A second chart then provides the systemic osmotic pressure of blood (or plasma) for the osmotically equivalent concentration of standard serum protein and also the osmotic pressure of the blood concentrated in the glomerulus by ultrafiltration to the degree indicated by the inulin-to-diodrast clearance ratio. These two values simplify and improve the calculation of renal resistance to blood flow.

A chart for blood viscosity, as affected by the serum protein concentration and the hematocrit, permits the ready calculation of renal resistance with allowance for the viscosity of the subject's blood. 
While the charts for osmotic pressure and viscosity of blood have been designed primarily to aid in studying renal function, they have wider applicability wherever blood viscosity and osmotic pressure are required without direct measurement.

\section{BIBLIOGRAPHY}

1. Lamport, H., Formulae for afferent and efferent arteriolar resistance in the human kidney; an application to the effects of spinal anesthesia. J. Clin. Invest., $1941,20,535$.

2. Smith, H. W., The Physiology of the Kidney. Oxford University Press, New York, 1937.

3. Smith, H. W., et al., Glomerular dynamics in normal human kidney. J. Clin. Invest., 1940, 19, 751.

4. Adair, G. S., Adair, M. E., and Greaves, R. I. N., Osmotic pressure, after re-solution, of serum, dried from frozen state (F. D. serum). J. Hyg., 1940, $40,548$.

5. Whittaker, S. R. F., and Winton, F. R., Apparent viscosity of blood flowing in isolated hindlimb of dog, and its variation with corpuscular concentration. J. Physiol., 1933, 78, 339.

6. Bingham, E. C., Fluidity and Plasticity. McGrawHill Book Co., New York, 1922.

7. Houwink, R., Elasticity, Plasticity and Structure of Matter. Macmillan Co., New York, 1937.

8. Shannon, J. A., Kidney. Ann. Rev. Physiol., 1942, 4, 297.

9. Lamport, H., The effects on renal resistance to blood flow of renin, angiotonin, pitressin and atropine, hypertension, and toxemia of pregnancy. J. Clin. Invest., 1942, 21, 685.

10. Wies, C. H., and Peters, J. P., Osmotic pressure of proteins in whole serum. J. Clin. Invest., 1937, 16, 93.

11. Lamport, $H$., The relative changes in afferent and efferent arteriolar resistance in the normal human kidney. J. Clin. Invest., 1941, 20, 545.

12. Lamport, H., Charts for osmotic pressure of blood. Applications of formulas for renal arteriolar resistance. Fed. Proc., 1942, 1, April. 